\title{
Excitation of Bulk Spin Waves by Acoustic Wave at the Plane Defect of a Ferromagnet
}

\author{
Y. Gusieva ${ }^{a, *}$, P. GraczyK ${ }^{b}$, O. Gorobets ${ }^{a, c}$ And M. KrawczyK ${ }^{b}$ \\ ${ }^{a}$ National Technical University of Ukraine "Igor Sikorsky Kyiv Polytechnic Institute", \\ 37 Peremogy ave., 03056, Kyiv, Ukraine,
}

${ }^{b}$ Faculty of Physics, Adam Mickiewicz University in Poznan, Umultowska 85, Poznań, 61-614, Poland, ${ }^{c}$ Institute of Magnetism, National Academy of Sciences of Ukraine, 36-b Vernadskogost., 03142, Kyiv, Ukraine

\begin{abstract}
Excitation of bulk spin waves by acoustic wave localized on the elastic planar defect in the bulk ferromagnet was analytically and numerically investigated. We showed that besides magnetic oscillations forced by acoustic wave strain the resonance between Kosevich wave and bulk spin wave can occurs. For the frequency of the Kosevich wave far below the resonance frequency the amplitude of dynamic magnetization is negligible. For the frequency above the resonance the acoustic wave excites bulk spin wave of the same frequency but different absolute value of the wave vector.
\end{abstract}

DOI: 10.12693/APhysPolA.133.489

PACS/topics: 75.30 Ds, 75.78.- - n, 75.80.+q

\section{Introduction}

Surface acoustic waves are extensively investigated in seismology [1], bio-sensing [2, 3] and phononics [4]. Recently much attention is focused on the coupling between acoustic waves and spin waves (SW) [5-7]. Interactions between mechanical and magnetic degrees of freedom are interesting from fundamental point of view and also for application as spin wave generators, transducers or filters in magnonic devices. Since magnonic devices, which operates with SW are mainly based on thin film technology, it is desirable to consider acoustic waves that are localized at the surface or interface [5-7]. Dynamic components of magnetization cause deformation of a solid due to magnetostriction. On the other hand, acoustic deformation induces the dynamic effective magnetic field that acts to magnetization via inverse magnetostriction. If the frequency and wavelength of this induced deformation (effective field) matches to the frequency and wavevector of the acoustic wave (spin wave), the resonance criterion is satisfied and the energy transfer between SW and acoustic wave occurs. This phenomenon is widely used in electromagnetism, recently also in magnonics for directional couplers [12-14]. In a thin layer - substrate system, in general, two kinds of surface acoustic waves can propagate. The Rayleigh wave is polarized in the sagittal plane, while the Love wave is a shear horizontal wave. The detailed description of surface waves and their existence limits are available in Ref. [8]. As comes from the symmetry considerations of coupled LandauLifshitz (LL) and wave motion equations, Rayleigh wave couples linearly to the forward volume SW, while Love wave couples linearly to the Damon-Eshbach mode. In the long-wavelength limit, the Love wave can be approx-

*corresponding author; e-mail: yuleva1313@gmail.com imated by the solution given by Kosevich [9] for a shear horizontal wave localized at the planar defect. We will further refer to this localized wave as a "Kosevich wave" (KAW).

We will consider the planar defect as a layer of finite thickness with different elastic properties and with transverse acoustic velocity much lower than the surrounding material. We will study analytically and numerically the influence of externally excited KAW on the dynamics of magnetization in the ferromagnetic solid. We present the solution for the magnetic oscillations forced by the acoustic wave in ferromagnetic material with homogeneous magnetic properties. These oscillations are solely bounded to the acoustic wave and thus, contrary to the resonance with the bulk SW, do not extend beyond the region of the existence of acoustic wave.

\section{Analytical model}

We consider a system of two semi-infinite ferromagnets, which are in contact along the plane $x O y$. The interface between the ferromagnets has finite thickness $\delta$ and it will be considered later on as a planar defect only for acoustic waves. Each material is characterized by the parameters of the exchange interaction $\alpha$, and the uniaxial magnetic anisotropy $\beta$.

The total magnetic energy of the system of two ferromagnetic semi-infinite media (the model of two-sublattice material) is:

$$
\begin{aligned}
W & =S \int \mathrm{d} z\left[A(z)_{i j} \boldsymbol{M}_{i} \boldsymbol{M}_{j}-\boldsymbol{H}_{0} \boldsymbol{M}_{i}\right. \\
& \left.+\frac{\alpha_{i}(z)\left(\partial_{z} \boldsymbol{M}_{i}\right)^{2}-\beta_{i}(z)\left(\boldsymbol{M}_{i} \boldsymbol{n}_{i}\right) \boldsymbol{n}_{i}}{2}\right],
\end{aligned}
$$

where $\boldsymbol{M}_{i(j)}$ are the magnetization vector of the $i, j$-th media $(i j=1,2, i \neq j), \boldsymbol{H}_{0}$ is external magnetic field which is parallel to the axis $O z, S$ is the area of the interface between two ferromagnets, $A_{i j}(z)$ is the exchange interaction between sublattices, $a_{i}(z)$ is the parameter of non-uniform exchange, $\beta_{i}(z)$ is the uniaxial magnetic 
anisotropie, the corresponding vector $\boldsymbol{n}_{i}$ is the unit vector of the uniaxial magnetic anisotropy axes, and $w_{\text {elast }}$ is the density of the magnetoelastic energy. Let's consider the magnetically homogeneous system with equal magnetic parameters, i.e., $\left.\mid M_{01}\right\rfloor=\left\lfloor M_{01}\right\rfloor=M_{0}, \alpha=$ $\alpha_{1}(z)=\alpha_{2}(z)$ and $\beta=\beta_{1}(z)=\beta_{2}(z)$, extended also on the interface, i.e., we will treat the whole system as a homogeneous media for SWs.

The solution of the wave equation for KAW is [9]:

$$
u_{y}=u_{0} \mathrm{e}^{-\kappa|z|} \sin (k x-\omega t)
$$

and its dispersion relation is: $\omega^{2}=\left(k^{2}-\kappa^{2}\right) v^{2}$. Here $u_{0}, k$ and $w$ are amplitude, wave number and angular frequency of the KAW, respectively, $v=\sqrt{\mu / \rho}$ is the velocity of acoustic shear wave. Parameter $\kappa$ is given by: $\kappa=\frac{1}{2} h k^{2}\left[\left(\mu-\mu_{s}\right) / \mu_{s}-\left(\rho-\rho_{s}\right) / \rho\right]$ and describes decay of the wave along the $z$ axis. Here $\mu$ is elastic moduli of the host material, $\mu_{s}$ - the elastic moduli of the planar defect layer, $\rho$ - the mass density of the host material, $\rho_{s}$ - the mass density on the planar defect. KAW has the phase velocity $c^{2}=\left(k^{2}-\kappa^{2}\right) v^{2} / k^{2}$.

Magnetoelastic energy density with KAW is: $w_{\text {elast }}=$ $2 B M_{0}\left(M_{x} S_{x z}+M_{y} S_{y z}\right)=-\kappa B / M_{0} u_{y} M_{y}$, where $B$ is the magnetoelastic coefficient and $S_{i j}$ is the strain tensor.

The corresponding effective magnetic field is given by:

$$
\begin{gathered}
\boldsymbol{H}_{e f}=-\delta W / \delta \boldsymbol{M}=-A(z) \boldsymbol{M}+\partial_{z}\left(\alpha_{1}(z) \partial_{z} \boldsymbol{M}\right) \\
+\beta(z)(\boldsymbol{M n}) \boldsymbol{n}-\boldsymbol{H}_{0}+\boldsymbol{e}_{y} \kappa B u_{y} / M_{0}
\end{gathered}
$$

To take into account magnetostatic interaction we introduce dynamic magnetostatic field: $\boldsymbol{h}=\boldsymbol{h}^{(m)}=$ $h_{x} \boldsymbol{e}_{x}-4 \pi \hat{N} \boldsymbol{m}$, where $\hat{N}$ is a tensor of the demagnetization coefficients in the plane $y O z$.

The linearized LL equation describing the dynamics of the perturbation magnetization $\boldsymbol{m}$ in the magnetically homogeneous system with magnetoelastic interaction takes the form [10]:

$$
\begin{aligned}
& \dot{\boldsymbol{m}} / g=\boldsymbol{M}_{0} \times(\alpha \Delta \boldsymbol{m}+\boldsymbol{h}+\beta(\boldsymbol{m} \boldsymbol{n}) \boldsymbol{n}) \\
& \quad-\left[\boldsymbol{M}_{0} \boldsymbol{H}_{0}^{i}+\beta\left(\boldsymbol{M}_{0} \boldsymbol{n}\right)^{2}\right] \frac{\boldsymbol{m}}{M_{0}^{2}}+|\kappa| B / M_{0} u_{x} \boldsymbol{e}_{y},
\end{aligned}
$$

where $\boldsymbol{H}_{0}^{(i)}$ is the internal static magnetic field. We assume the solution of Eq. (4) in the form: $\boldsymbol{m}=$ $\boldsymbol{m}_{0}(\xi) \mathrm{e}^{-\kappa|z|}, \xi=k x-\omega t$, which substituted to (4) yields:

$$
\begin{aligned}
& -\omega \partial_{\xi} \boldsymbol{m}_{0}=g \boldsymbol{M}_{0} \times\left[\alpha k^{2} \partial_{\xi} \boldsymbol{m}_{0}-4 \pi \hat{N} \boldsymbol{m}_{0}\right. \\
& \left.+\left(\alpha \kappa^{2}-H_{0}^{(i)} / M_{0}-\beta\right) \boldsymbol{m}_{0}+\kappa B u_{0} / M_{0} \sin \xi \boldsymbol{e}_{y}\right]
\end{aligned}
$$

with: $f_{0}=\kappa B u_{0} / M_{0}, \gamma_{0}=\alpha \kappa^{2}-H_{0}^{(i)} / M_{0}-\beta$.

The Eq. (5) can be transformed to two scalar equations for two functions $m_{0, y}$ and $m_{0, z}$ :

$$
\begin{aligned}
& -\omega \partial_{\xi} m_{0 y}=g M_{0}\left(\alpha k^{2} \partial_{\xi}^{2} m_{0 y}+\gamma_{0} m_{0 z}-4 \pi N_{z z} m_{0 z}\right) \\
& -\omega \partial_{\xi} m_{0 z}=g M_{0}\left(\alpha k^{2} \partial_{\xi}^{2} m_{0 y}+\left(\gamma_{0}-4 \pi N_{y y}\right) m_{0 y}\right. \\
& \left.\quad+f_{0} \sin \xi\right) .
\end{aligned}
$$

Solving the system of Eqs. (6), (7) and using magnetostatic equations for determination tensor components $N_{y y}=0, N_{z z}=\kappa^{2} /\left(\kappa^{2}-k^{2}\right)$ we get the following solutions of the LL equation:

$$
\begin{aligned}
& m_{y}=f_{0} \Omega_{z} u_{y} /\left(\Omega_{y} \Omega_{z}-a^{2}\right) \\
& m_{z}=-f_{0} a \sqrt{1-u_{y}^{2} /\left(\Omega_{y} \Omega_{z}-a^{2}\right)},
\end{aligned}
$$

where $a=\omega / g M_{0}, \Omega_{z}=\alpha k^{2}-\gamma_{0}+4 \pi \kappa^{2} /\left(\kappa^{2}-k^{2}\right)$, $f_{0}=\kappa B u_{0} / M_{0}, \gamma_{0}=a \kappa^{2}-\frac{H_{0}^{i}}{M_{0}}-\beta, \Omega_{y}=a k^{2}-\beta$.

Using solutions (8) and (9) we estimate the ratio of the magnetic energy $W$ to the elastic energy $E_{a}$ for parameters of Co: $H_{0}=10^{5} \mathrm{~A} / \mathrm{m}, m_{s}=10^{3} \mathrm{~Pa}$, $r_{S}=4000 \mathrm{~kg} / \mathrm{m}^{3}, h=1 \mathrm{~nm}, r_{S}=8900 \mathrm{~kg} / \mathrm{m}^{3}$, $M_{0}=10^{6} \mathrm{~A} / \mathrm{m}, a=2 A / M_{0}^{2}, B=6.5 \times 10^{6} \mathrm{~J} / \mathrm{m}^{3}$, $g=176 \mathrm{GHz} / \mathrm{T}, c_{44}=80 \mathrm{GPa}$, for simplicity we neglect magnetic anisotropy $b=0$ :

$$
W / E_{a}=\frac{\alpha B^{2} \kappa\left(\Omega_{z}^{2}+a^{2}\right)}{2 c_{44} M_{0}^{2}\left(\Omega_{y} \Omega_{z}-a^{2}\right)^{2}}
$$

For arbitrary wave number of the KAW, the magnetic energy is negligible as compared to $E_{a}$. However, if the KAW is in resonance with the SW, i.e. when:

$$
\omega=\alpha k^{2}+H_{0} / M_{0}
$$

the magnetic energy is of the same order as the acoustic energy. The interface acoustic wave excites the SW in the region of their existence. This case will be shown in the next section in more detail by numerical simulations.

\section{Numerical simulations}

In this section we discuss numerical results for KAW in Co, taking into account only external magnetic field and exchange interaction in the LL equations. Fig. 1 shows the dispersion relation of bulk transverse acoustic wave and of bulk SW in Co. The values used in calculation are listed in section 2 . The violet curve with blank squares indicates dispersion of KAW whereas the red circles indicates dispersion for the bulk SW. The amplitude of $\boldsymbol{m}$ in Eqs. (8), (9) is significant if Eq. (10) is satisfied. It is equivalent to the condition of the resonance between KAW and bulk SW, which occurs at the point of the crossing, i.e. for $f_{1}=3.6 \mathrm{GHz}, k_{1}=9.4 \mu \mathrm{m}^{-1}$, for $f_{2}=4.14 \mathrm{GHz}, k_{2}=23.6 \mu \mathrm{m}^{-1}$.

The simulations by finite-element method were performed by solving LL equations in the form $\dot{m}_{y}=$ $\omega_{0} m_{z}-\alpha \nabla^{2} m_{z}, \dot{m}_{z}=-\omega_{0} m_{y}+\alpha \nabla^{2} m_{y}-g B \partial_{z} u_{y}$, where $\omega_{0}=g \mu_{0} H_{0}$ and the KAW is described by Eq. (2). We investigated the time dependent evolution of magnetization dynamics for the KAW of the frequencies indicated by black points in Fig. 1, i.e. at the frequency below the resonance $f_{2}$, at the frequency above the resonance $f_{2}$, and at bulk SW-KAW dispersion crossing $\left(k_{2}, f_{2}\right)$.

In Fig. 2 the magnetization component $m_{z}$ after 3 ns of excitation by Kosevich wave at the defect layer is shown. For the frequency of KAW far below the resonance (Fig. 2a) the values of $m_{z}$ are neglible since that are only SW oscillations forced by the acoustic wave. At the frequency of $4.14 \mathrm{GHz}$ (Fig. 2b) the bulk SW of sig- 


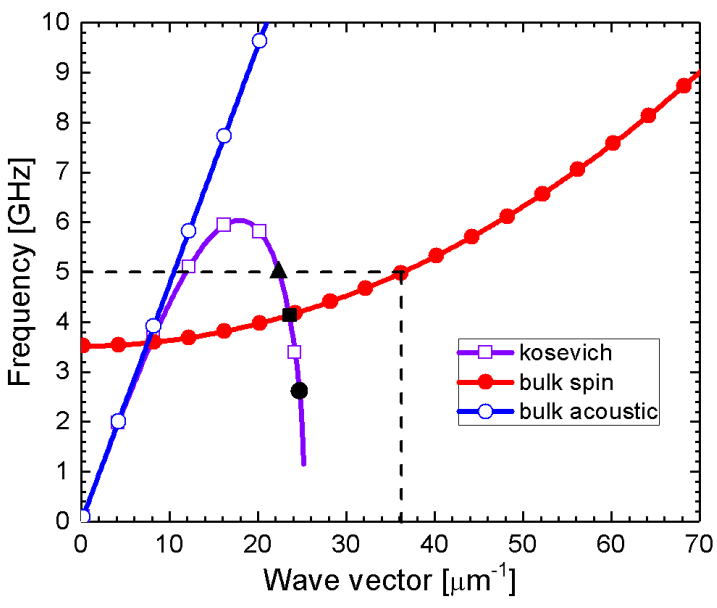

Fig. 1. Dispersion relation for bulk SW (filled circles), transverse acoustic wave (circles) and KAW (squares) in Co.

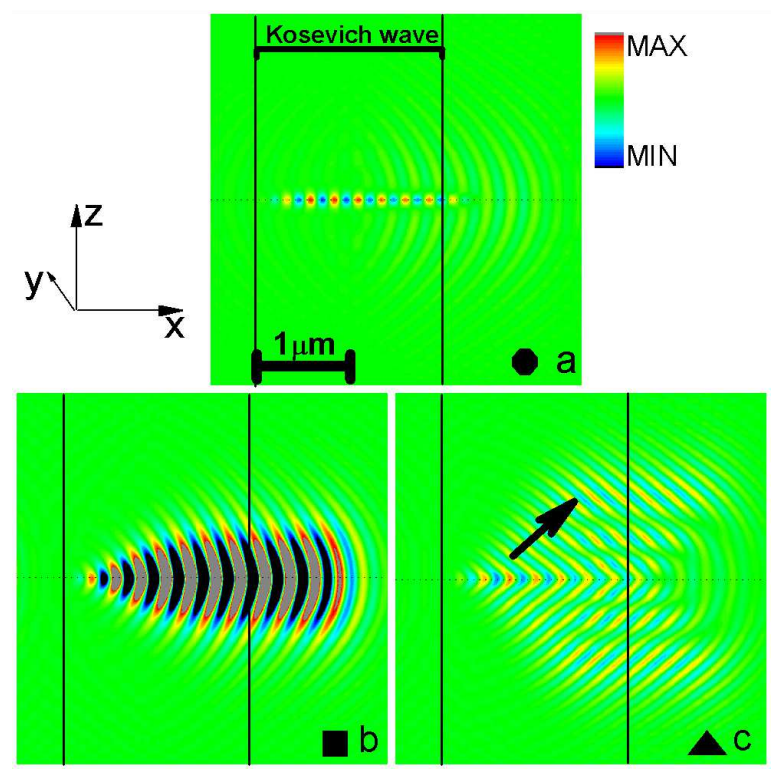

Fig. 2. The value of $m_{z}$ after $3 \mathrm{~ns}$ from the excitation by KAW at the area indicated by vertical solid lines for the frequencies (a) $2.8 \mathrm{GHz}$, (b) $4.14 \mathrm{GHz}$ and (c) $5 \mathrm{GHz}$. Dotted line marks the defect plane. The relative scale of the amplitude between figures is preserved.

nificant amplitude is excited, which spreads into the ferromagnetic medium. In this case the criterion of the resonance (Eq. (10)) is satisfied. For the frequency above the resonance, besides forcing magnetic oscillations, KAW excites bulk SW of the same frequency but different absolute value of the wave vector. The mechanism of the excitation is indicated in Fig. 1 by black dashed lines. The wave vector of KAW matches with the component of the spin wave vector which describes SW propagating in the direction outside of the interface (see arrow in Fig. 2). Since the phase matching criterion is satisfied only for one component of the wave vector, the resonance is not complete. Acoustic wave at the interface excites the wave until it becomes decoupled. Then, it starts to couple again. In consequence, as it is visible in Fig. 2c, SW forms multiple separated "beams" of wave fronts.

\section{Conclusions}

In this paper we demonstrate a new possibility to excite bulk spin wave by acoustic wave in the system of the ferromagnetic material with the planar defect for elastic waves. The significant effect can be achieved if the resonance between KAW and SW occurs. The proposed mechanism may be further utilized to design planar or spot SW sources or acoustic wave-SW transducers [11] for the SW propagating inside of the bulk ferromagnetic material.

\section{Acknowledgments}

We acknowledge the financial assistance from the European Union's Horizon 2020 research and innovation programme under the Marie Skłodowska-Curie grant agreement No 644348 (MagIC) and by Polish National Science Center under grant no. UMO-2012/07/E/ST3/00538.

\section{References}

[1] S. Brule, E.H. Javelaud, S. Enoch, S. Guenneau, Phys. Rev. Lett. 112, 133901 (2014).

[2] A. Sagasti, J. Gutierrez, M. San Sebastian, J.M. Barandiaran, IEEE Trans. Magn. 53, 4 (2017).

[3] J.-T. Luo, A.-J. Quan, G.-X. Liang, Z.-H. Zheng, S. Ramadan, C.Fu, H.-L. Li, Y.-Q. Fu, Ultrasonics 75, 63 (2017).

[4] M. Maldovan, Nature 503, 209 (2013).

[5] P.G. Gowtham, T. Moriyama, D.C. Ralph, R.A. Buhrman, J. Appl. Phys. 118, 233910 (2015).

[6] P. Graczyk, J. Kłos, M. Krawczyk, Phys. Rev. B 95, 104425 (2017).

[7] J. Janušonis, C.L. Chang, P.H.M. van Loosdrecht, R.I. Tobey, Appl. Phys. Lett. 106, 181601 (2015).

[8] G.W. Farnell, E.L. Adler, Physical Acoustic, Vol. 9, Academic Press, 1972.

[9] A.M. Kosevich, A.V. Tutov, Phys. Lett. A 213, 265 (1996).

[10] I. Akhiezer, V.G. Bar'yakhtar, S.V. Peletminskii, Spin Waves, Interscience, New York 1968.

[11] A. Kamra, H. Keshtgar, P. Yan, G.E.W. Bauer, Phys. Rev. B 91, 104409 (2015).

[12] A.V. Sadovnikov et al., Appl. Phys. Lett. 107, 202405 (2015).

[13] A.V. Sadovnikov, et al., Phys. Rev. Appl. 7, 014013 (2017).

[14] A.V. Sadovnikov et al., IEEE Trans. Magn. 53, 1 (2017). 